\title{
Dual-Energy Contrast-Enhanced Digital Spectral Mammography
}

National Cancer Institute

\section{Source}

National Cancer Institute. Dual-Energy Contrast-Enhanced Digital Spectral

Mammography. NCI Thesaurus. Code C119679.

A technique that uses high-energy and low-energy digital mammography with administration of an iodinated contrast agent. The differences between X-ray attenuation of iodine and breast tissues at these two energy levels are used to suppress the background breast tissue. This technique is useful in identifying lesions in dense breasts that are not visible using standard mammography. 